\title{
Hot electron excitation from titanium nitride using
}

\section{visible light}

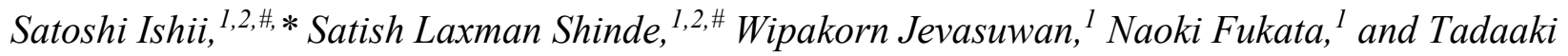
$\operatorname{Nagao}^{1,2}$

${ }^{1}$ International Center for Materials Nanoarchitectonics (MANA), National Institute for Materials Science (NIMS), Tsukuba, Ibaraki 305-0044, Japan

${ }^{2}$ Core Research for Evolutional Science and Technology (CREST), Japan Science and Technology Agency (JST), Kawaguchi, Saitama, 332-0012 Japan

\# These authors contributed equally.

Table R1 Sputtering conditions to deposit TiN, ZnO, and Au films.

\begin{tabular}{|l|l|l|l|}
\hline & TiN & $\mathrm{ZnO}$ & $\mathrm{Au}$ \\
\hline DC or RF & $\mathrm{DC}$ & $\mathrm{RF}$ & $\mathrm{DC}$ \\
\hline target & $\mathrm{TiN}$ & $\mathrm{ZnO}$ & $\mathrm{Au}$ \\
\hline power $[\mathrm{W}]$ & 100 & 200 & 100 \\
\hline gas $[\mathrm{SCCM}]$ & $\mathrm{Ar} / 20$ & $\mathrm{Ar} / 18, \mathrm{O}_{2} / 2$ & $\mathrm{Ar} / 20$ \\
\hline pressure during sputtering $[\mathrm{Pa}]$ & 0.48 & 0.48 & 0.48 \\
\hline substrate-to-target distance $[\mathrm{mm}]$ & 85 & 85 & 85 \\
\hline
\end{tabular}




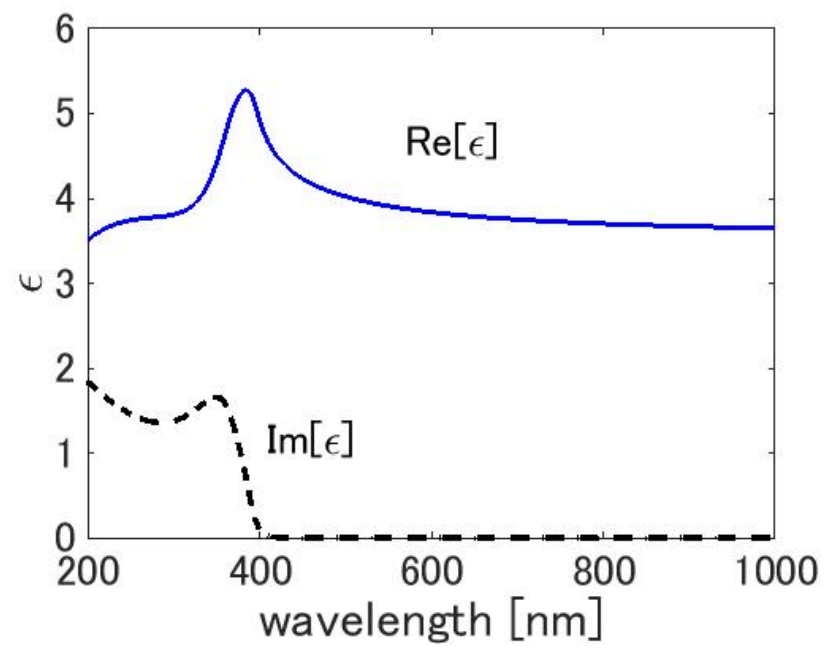

Figure S1. Retrieved complex permittivity of the sputtered zinc oxide film measured by a spectroscopic ellipsometry.

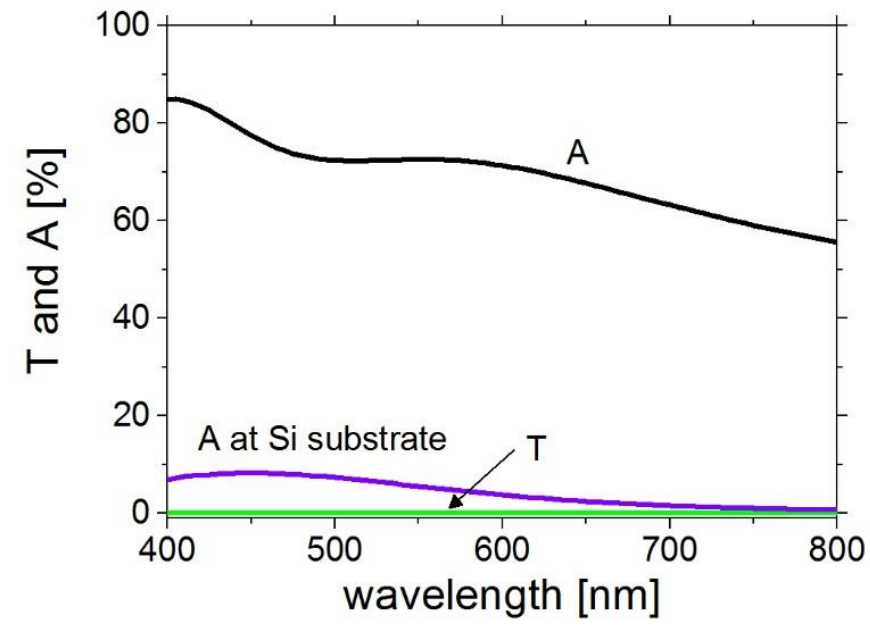

Figure S2. Analytically calculated transmittance (T), total absorptivity (A) and absorptivity of the silicon substrate for the TiN-MIM where the thickness of zinc oxide is $20 \mathrm{~nm}$. 

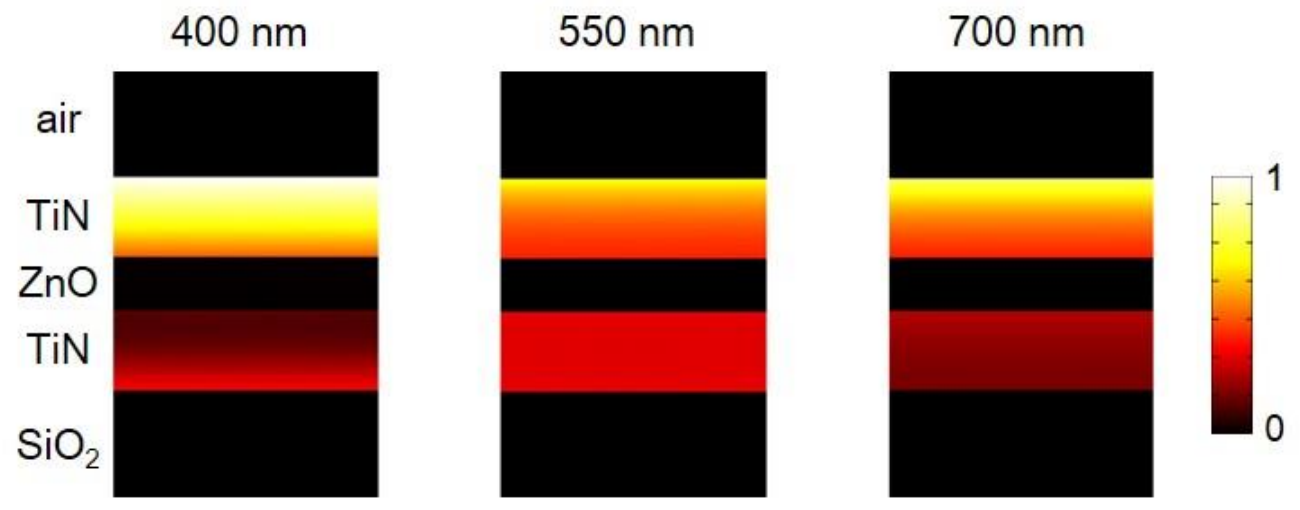

Figure S3. Analytically calculated normalized absorptivity cross sections of the TiN-MIM having a $20 \mathrm{~nm}$ thick zinc oxide simulated at 400, 550, and $700 \mathrm{~nm}$ in wavelength.
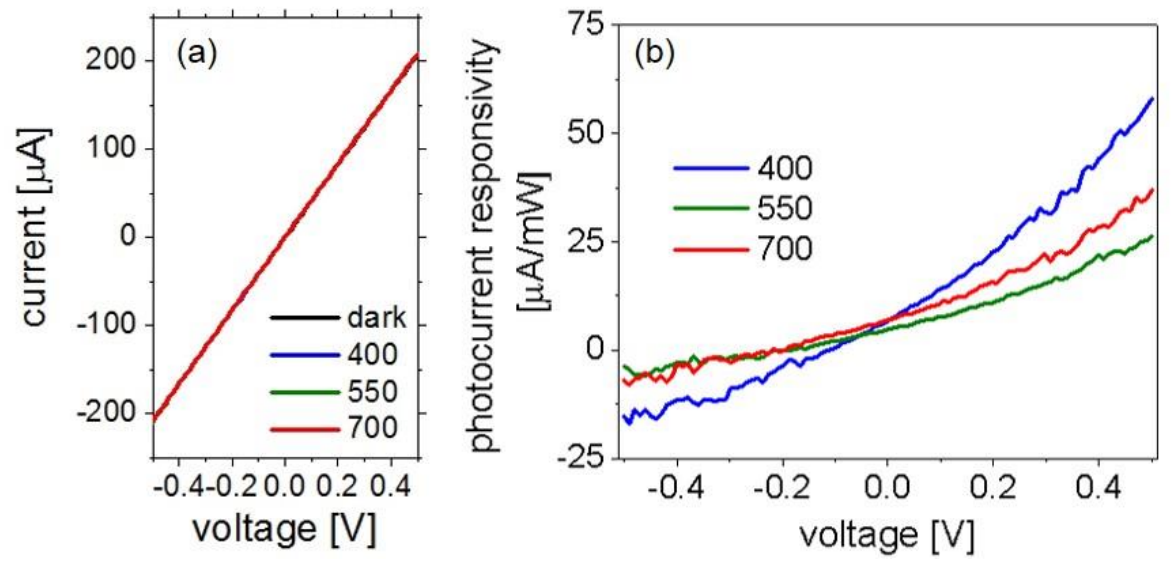

Figure S4. (a) I-V curves of the TiN-MIM (20 nm thick zinc oxide) sample under light illumination at 400, 550, and $700 \mathrm{~nm}$ in wavelength and at dark. The I-V curve measured at dark is identical to the plot shown in Figure 2(a). (b) Photocurrent responsivity calculated from sub-figure (a). 
(a)

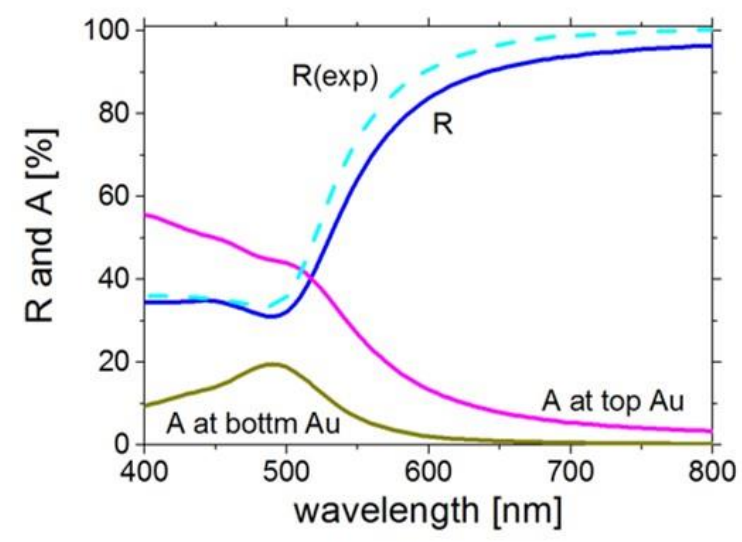

(b)

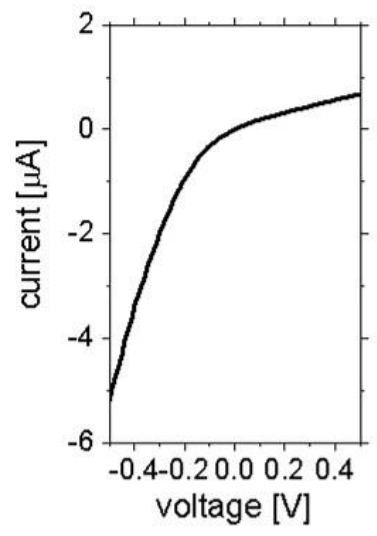

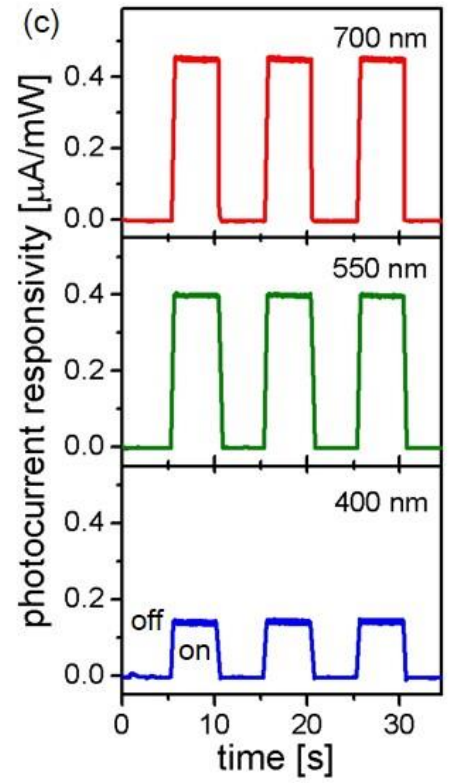

Figure S5. Optical and electrical characterization of the Au-MIM where the zinc oxide thickness is $20 \mathrm{~nm}$. (a) Analytically calculated reflection (R), absorptivity (A) at the top Au, and A at the bottom Au. The dashed line shows the measured reflectance (R(exp)). (b) I-V curve at dark. (c) Time-dependent photocurrent measured at 400, 550, and $700 \mathrm{~nm}$ in wavelength at zero bias. The light source was turned on and off every five seconds and photocurrent is seen only when the light is on. 